\title{
ON THE CALCUIATION OF
}

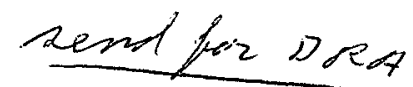

\section{THREL-DIMENSIONAL LAMINAR BOUNDARY LAYER FLOWS}

J. A. Fillo*, R. Burbank ${ }^{\dagger}$

University of Massachusetts, mherst, Massachusetts

NOMENCLATUPE
$a=c y l i n d e r$ radius
$f=\int_{0}^{n} F(\xi, \lambda, \zeta) d \lambda$
$F=u / U$
$g=\int_{0}^{n} G(\xi, \lambda, \zeta) d \lambda$
$G=W / H$
$p=$ pressure
$U=x$-component of inviscid flow
$W=z$-comporent of inviscid flow
$\mathrm{u}_{\infty}=$ velocity upstream of the flat plate
$u, v, w=v e l o c i t y$ components in the $x, y, z$ directions
$\bar{x}$ distance from cylinder axis to the leading edge of the flat plate $(=0.5 .7 \mathrm{~cm})$

$x, y, z=$ Cariesian coordinates

$\xi, \eta, \zeta=$ transfomed coordinates

$\lambda=$ cumy variable of inierration

$\psi, \phi=$ streanfunctions

$\rho=$ density

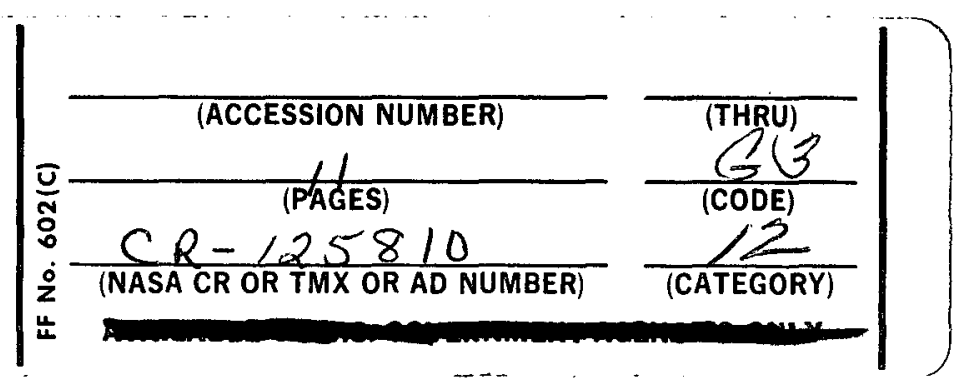

* Associate Professor, Cepartment of Hechanicai and Aerospace Engincering

TGraduate Pesearci Mssistarit, Depariment of Mechanical and Aerospace Engineering 


$$
\begin{aligned}
& \beta=2 \xi / U U_{\xi} \\
& \beta^{*}=2 \xi / U W_{\zeta} \\
& K=2 \xi / U^{2} U_{\zeta} \\
& K^{*}=2 \xi / W W_{\zeta} \\
& \tau_{x y}{ }^{\top} z y=\text { shear stress coriponents } \\
& H^{\prime}=\text { dynamic viscosity } \\
& \nu=\text { kinematic viscosity }
\end{aligned}
$$

Subscripts

$x, y, z, n, \ldots=$ differentiation (except on $\tau$ )

\section{INTRODUCTION}

In a recent paper, Wang ${ }^{1}$ introduces an approximation which reciuces the computation of three-dimensional, laminar, compressible, boundarylayer equations to the problem of solving two-dimensional type boundary layer equations. Assuming familiarity with Wang's work, it is to be noted that the test case chosen by Wang had been previously studied by Fannel op ${ }^{2}$ who had introduced a small cross-flow perturbation method. The perturbation method itself reduced the computation of the threedimensional boundary layer equations to that of two-dimensional type bouridary layer equations. While on the one hand Wang's results compare favorably with Fannelop's, it would seem that a more stringent test of Wang's miethod would be the comparison with a fully three-dimensional boundary layer calculation. In this note we provide such a comparison with the results from our three-dimensional calculations. In addition we consider another aspect of Vlang's approximation for the calculation of three-dimensional flows. 
The test case which we have chosen is incompressible, laminar flow past a flat plate with attached cylinder (sce Fig. 1). The boundary layer of interest is formed on the plate upstream of the cylinder. This problem has been studied by Sowerby ${ }^{3}$ wi h a Blasius-type series solution and by Dwyer ${ }^{4}$ with a firite difference method. Our method differs from Dwyer's in that we introduce two stream functions such that the continuity equation is satisfied.

\section{ANALYSIS}

The governing boundary layer equations, written in Cartesian coordinates are

$$
\begin{aligned}
& u_{x}+v_{y}+w_{z}=0 \\
& u u_{x}+v u_{y}+w u_{z}=-1 / p p_{x}+v u_{y y} \\
& u w_{x}+w w_{y}+w w_{z}=-1 / p p_{z}+v w_{y y}
\end{aligned}
$$

subject to boundary conditions

$$
y=0: u=v=w=0, y=\infty: u=U, w=w
$$

where $-1 / \rho p_{x}=U U_{x}+W U_{z},-1 / \rho p_{z}=U U_{x}+W W_{z}$

and

$$
U=U_{\infty}\left\{1+\frac{z^{2}-(x-\bar{x})^{2}}{\left(1 / a^{2}\right)\left[(x-\bar{x})^{2}+z^{2}\right]^{2}}\right\} ; W=-\frac{2 U_{\infty} z(x-\bar{x})}{\left(1 / a^{2}\right)\left[(x-\bar{x})^{2}+z^{2}\right]^{2}}
$$

for the present problem under investigation.

Introciucing a $B$ lasius-type transforration, $n=(U / 2 v x)^{\frac{1}{2}} y, \xi=x$ and $\zeta=z$ as well as stream functions

$$
u=\psi_{y}, v=-\psi_{x}-\phi_{z}, w=\phi_{y}
$$


where

$$
\psi(x, y, z) \equiv(2 v x U)^{\frac{1}{2}} f(\xi, \eta, \zeta) ; \phi(x, y, z) \equiv W\left(\frac{2 v x}{U}\right)^{\frac{1}{2}} g(\xi, \eta, \zeta)
$$

Eqs. (2) and (3) beconie

$$
\begin{aligned}
F_{\eta n} & +(1+\beta / 2) f F_{\eta}+\beta\left(1-F^{2}\right)+K(1-F G)+\left(\beta^{*}-K / 2\right) g F_{\eta} \\
& +2 \xi\left(f_{\xi} F_{\eta}-F F_{\xi}\right)+2 \xi \frac{W}{U}\left(g_{\zeta} F_{\eta}-G F_{\zeta}\right)=0 \\
G_{n \eta} & +(1+\beta / 2) f G_{\eta}-\beta\left(1-G^{2}\right)+K^{*}(1-G F)+\left(\beta^{*}-K / 2\right) g G_{n} \\
& +2 \xi\left(f_{\xi} G_{n}-F G_{\xi}\right)+2 \xi \frac{W}{U}\left(g_{\zeta} G_{\eta}-G G_{\zeta}\right)=-\tilde{0}
\end{aligned}
$$

with the boundary conditions at $n=0: f=F=G=g=0$ and at $\eta=\infty: F=1, G=1$.

To integrate Eqs. (9) and (10) two initial conditions are needed, and , these are deduced by considering two limiting cases of Eqs. (9) and (10): (a) $\xi=0, \zeta \neq 0$; (b) $\xi \neq 0, \zeta=0$. Eqs. (9) and (10), for condition (a), reduce to Blasius equations. Referring to Fig. (1) condition (b) is the "line of symmetry" condition. Along this line $w=0$ as well as $\%$. In other words $w / W=G$ is indeterminate. On the other hand, ${ }_{z}$ and $H_{z}$ are not zero along $z=0$ so that by applying L'Hopital's rule to $\mathrm{w} / \mathrm{W}$, $G=W_{\zeta} / W_{\zeta}$ along $\zeta=0$. Consequently the equations for condition (b) are determined from Eqs. (9) and (10) by simply letting $\zeta$ go to zero.

To solve Eqs. (9). and (10) as well as the equations resulting from the initial conditions, an implicit finite difference procedure of Crank-Nicholson type is applied. The resulting non-linear equations are replaced initially by linear difference equations. An iteration procedure is used until the non-linear difference equations corresponding to the grid points employed have been solved. Calculations proceeded in the downstrean direction, for a given $z$-station, until flow reversal appeared 
in the u-component of velocity. Step sizes used in the calculations are: $\Delta \xi=\Delta \zeta=.61, \Delta n=.20$.

In order to check Wang's approximation against the full threedimensional calculation, we must simplify E.qs. (9) and (10) accordingly:We replace derivatives in the crossflow direction, i.e., the $\zeta$ derivatives, by their values at the edge of the inviscid flow, thereby reducing Eqs. (9) and (10) to quasi-two dimensional equations. Therefore, the following relations will be used:

$$
\begin{aligned}
G F_{\zeta} & =G\left(1 / U U_{\zeta}-1 / U U_{\zeta} F\right) \sim G(1-F) 1 / U U_{\zeta}, G G_{\zeta}=G\left(\frac{1}{W} W_{\zeta}-\frac{1}{W} W_{\zeta} G\right) \\
& \sim G(1-G) \frac{1}{W} W_{\zeta} ; g_{\zeta}=0
\end{aligned}
$$

Insofar as shear stress data and flow reversal are of iniportance, Tables 1 and 2 are a comparison between the full and approximate threediniensional calculations of $F_{n}\left(=f_{n n}\right)$ and $G_{n}\left(=g_{n i n}\right)$ at $n=0$. These values are proportional to the shear stress at the surface since

$$
\begin{aligned}
\tau_{x y}=\left.\mu \frac{\partial u}{\partial y}\right|_{y=0} & =\mu U\left(\frac{U}{2 v x}\right)^{\frac{1}{2}} f_{n n}(\xi, 0, \zeta), \tau_{z y}=\mu \frac{\partial W}{\partial y}:_{y=0} \\
& =\mu W\left(\frac{U}{2 v x}\right)^{\frac{1}{2}} g_{n n}(\xi, 0, \zeta)
\end{aligned}
$$

Fig. (1) contains the flow reversal line as determined by both methods. Up to a $z$ or $\zeta$ value of 6.10 , the full and approximate three-dimensional calculations predict flow reversal in the u-component, the approximate method predicting flow reversal further downstream from the leading edge than the full three-dimensional calculations. At $\zeta=7.32$, the full three-dimensional calculations predict flow reversal while the approximate method does not. By the approximate method, $F_{\eta}(0)$ (or $f_{n \eta}(0)$ ) reaches a 
minimum and then increases with $x$ or $\xi$, the minimum point shifting to smaller $\xi$ values as $\zeta$ increases. The calculations of $G_{n}(0)$ by both methods, and for all $\zeta$ values, even in the region where the approximate

- method fails to predict flow reversal, show good agreement.

While in two-dimensional, incompressible flow, the necessary condition for flow reversal is an adverse pressure gradient, we find that for three-dimensional flows an adverse pressure gradient is not necessarily a necessary condition for flow reversal. As a matter of fact, for the present problem at $\zeta=9.05$ and flow reversal, the pressure gradient is favorable. As Dwyer ${ }^{4}$ noted, the flow reversal of the u-component of velocity is caused by crossflow effects near the bottom of the boundary layer. That is, flow reversal is driven by the convective term, $w u_{z}$, which is positive and overcomes the negative $p_{x}$ term. Consequently $w u_{z}$ is an important term, and in particular, $u_{z}$, insofar as flow reversal is concerned so that by approximating $u_{z}$ in certain regions of the flow field, $w u_{z}$ can take on values which do not balance properly with the pressure gradient term. This is the case at $\zeta=7.32$ - the quasi-two dimensional approximation breaks down as flow reversal is approacheci, although for a large range of $\xi$ values, the approximate values of $F_{\eta}(0)$ are in good agreement with the full threedimensional calculations. This is also true at $\zeta_{2}=9.15$.

At most, then, what one may conclude from these results, is that Wang's approximation may not be valid in some applications and, more likely, in some particular region of a flow field, for example, as discussed above. Where the method does not break down, agreenent is most satisfactory with the full calculations. 
To explore the possible extension of Wang's method for further research, we considered the following calculation: adopting the approximate method, Eq. (11), along the line $\zeta=3.05=$ constant, and using the subsequent results as initial conditions for the full threedimensional calculations, we find that at the next station, $\zeta=3.66$, flow reversal is predicted at the same $\xi$ value as that obtained by starting the calculations along the line of symmetry, $\zeta=0$. In other words, the approximate method may be of interest in calculating three-dimensional flows where a line of symietry is not present. The major difficulty in such flows are the initial conditions with respect to the crossflow derivatives and their calculation. The idea is to apply the approximate method along some initial line and then revert to the full three-dimensional calculations. This idea is being applied to other problemis as well.

\section{REFERENCES}

TWang, K. K., "An Effective Approximation for Conputing the ThreeDimensional Laminar Boundary-Layer Flows," AIAA Journal, Vol. 9, iNo. 8, Aug. 1971, pp. 1649-1651.

${ }^{2}$ Fannelop, T. K.; "A Method of Solving the Three-Dimensional Laminar Boundary Layer Equations with Application to a Lifting Reentry Body," AIAA Journà7, Vol. 6, No. 6, June 1968, pp. 1075-1084.

${ }^{3}$ Sowerby, L., "The Three-Dimensional Laminar Boundary Layer on a Flat Plate," Journal of Fluid Mechanics, Vol. 22, Pt. 3, 1965, pp. 587-598. ${ }^{4}$ Dwyer, H. A., "Solution of a Three-Dimensional Bounciary-Layer Flow with Separation," AIAA Journal, Vo1. 6, No. 7, July 1968, pp. 1336-1342. 


\section{TABLE 1}

COMPARISOIN BETWEEN FULL AND APPRDXIMATE

THREE-DIMENSIONAL BOUNDARY LAYER CALCULATIONS OF $F_{\eta}(0)$

$$
\zeta=3.05 \mathrm{~cm} . \quad \zeta=6.10 \mathrm{~cm} . \quad \zeta=7.32 \mathrm{~cm} . \quad \zeta=9.15 \mathrm{~cm} \text {. }
$$

६-cm. Full Approx. Fuli Approx. Full Approx. Full Approx.

\begin{tabular}{|c|c|c|c|c|c|c|c|c|}
\hline 0 & .4696 & .4696 & .4696 & .4696 & .4696 & .4696 & .4696 & .4695 \\
\hline .61 & .4677 & .4677 & .4677 & .4677 & .4678 & .4678 & .4678 & .4679 \\
\hline 3.66 & .4631 & .4631 & .4637 & .4637 & .4640 & .4640 & .4646 & .4646 \\
\hline 7.93 & .4508 & .4508 & .4528 & .4529 & .4539 & .4540 & .4558 & .4560 \\
\hline 10.37 & .4408 & .4408 & .4442 & .4444 & .4467 & .4464 & .4493 & .4496 \\
\hline 13.42 & .4230 & .4231 & .4296 & .4300 & .4331 & .4337 & .4389 & .4397 \\
\hline 17.69 & .3814 & .3818 & .3974 & .3989 & .4056 & .4075 & .4188 & .4213 \\
\hline 20.13 & .3417 & .3428 & .3691 & .3722 & .3827 & .3865 & .4039 & .4087 \\
\hline 23.79 & .2340 & .2393 & .3006 & .3120 & .3315 & .3444 & .3766 & .3906 \\
\hline 24.40 & .2057 & .2126 & .2839 & 2987 & .3798 & .3361 & .3715 & .3885 \\
\hline 25.01 & .1701 & .1811 & .2646 & .2843 & .3067 & .3275 & .3661 & .3869 \\
\hline 25.62 & .1250 & .7431 & .2714 & .2685 & .2916 & .3187 & .3605 & .3861 \\
\hline 26.23 & & .0946 & .2126 & .2512 & .2735 & .3097 & .3543 & .3863 \\
\hline 26.84 & & & .1685 & .2322 & .2495 & .3008 & .3471 & .3876 \\
\hline 27.45 & & & .0874 & .2173 & .2067 & .2919 & .3378 & .3906 \\
\hline 28.06 & & & & .1880 & .1041 & .2835 & .3209 & .3955 \\
\hline 28.67 & & & & .1617 & & .2759 & .2547 & .4028 \\
\hline 29.28 & & & & .1310 & & .2694 & & .4730 \\
\hline 29.89 & & & & .0926 & & .2646 & & .4269 \\
\hline 30.50 & & & & & & .2623 & & .4452 \\
\hline 31.11 & & & & & & .2632 & & .4687 \\
\hline
\end{tabular}


TABLE 2

COMPARISON BETWEEI FULL AND AFPROXIMATE

THREE-DIMENSIONAL BOUNDARY LAYER CALCULATIONS OF $G_{\eta}(0)$

$$
\zeta=3.05 \mathrm{~cm} . \quad \zeta=6.10 \mathrm{~cm} . \quad \zeta=7.32 \mathrm{~cm} . \quad \zeta=9.15 \mathrm{~cm} .
$$

छ-cm. Full Approx. Full Approx. Fuli Approx. Full Approx.

\begin{tabular}{lllllllll}
\hline 0 & .4696 & .4696 & .4696 & .4696 & .4696 & .4696 & .4696 & .4696 \\
.61 & .5309 & .5307 & .5298 & .5295 & .5292 & .5289 & .5280 & .5277 \\
3.66 & .8567 & .8555 & .8488 & .8477 & .8443 & .8432 & .8363 & .8353 \\
7.93 & 1.355 & 1.351 & 1.333 & 1.330 & 1.321 & 1.318 & 1.300 & 1.297 \\
10.37 & 1.669 & 1.664 & 1.637 & 1.632 & 1.618 & 1.614 & 1.586 & 1.582 \\
13.42 & 2.102 & 2.094 & 2.050 & 2.044 & 2.021 & 2.015 & 1.970 & 1.965 \\
17.69 & 2.809 & 2.798 & 2.713 & 2.703 & 2.661 & 2.650 & 2.569 & 2.559 \\
20.13 & 3.288 & 3.278 & 3.152 & 3.139 & 3.077 & 3.064 & 2.949 & 2.935 \\
23.79 & 4.173 & 4.172 & 3.928 & 3.909 & 3.797 & 3.774 & 3.580 & 3.552 \\
24.40 & 4.348 & 4.351 & 4.075 & 4.055 & 3.932 & 3.904 & 3.693 & 3.660 \\
25.01 & 4.534 & 4.543 & 4.230 & 4.207 & 4.071 & 4.039 & 3.809 & 3.770 \\
25.62 & 4.729 & 4.750 & 4.392 & 4.366 & 4.216 & 4.178 & 3.927 & 3.5 \\
26.23 & & 4.973 & 4.561 & 4.532 & 4.366 & 4.321 & 4.048 & 3.3 \\
26.84 & & & 4.723 & 4.707 & 4.520 & 4.469 & 4.171 & 4. \\
27.45 & & & 4.892 & 4.891 & 4.647 & 4.621 & 4.296 & 4.2 \\
28.06 & & & & 5.086 & 4.754 & 4.777 & 4.408 & 4.3 \\
28.67 & & & 5.293 & & 4.938 & 4.411 & $4.45 \delta$ \\
29.28 & & & 5.513 & & 5.102 & & 4.545 \\
29.89 & & & 5.747 & & 5.270 & & 4.648 \\
30.50 & & & & & & 5.441 & & 4.746 \\
31.11 & & & & & & 5.613 & & 4.837 \\
\hline
\end{tabular}




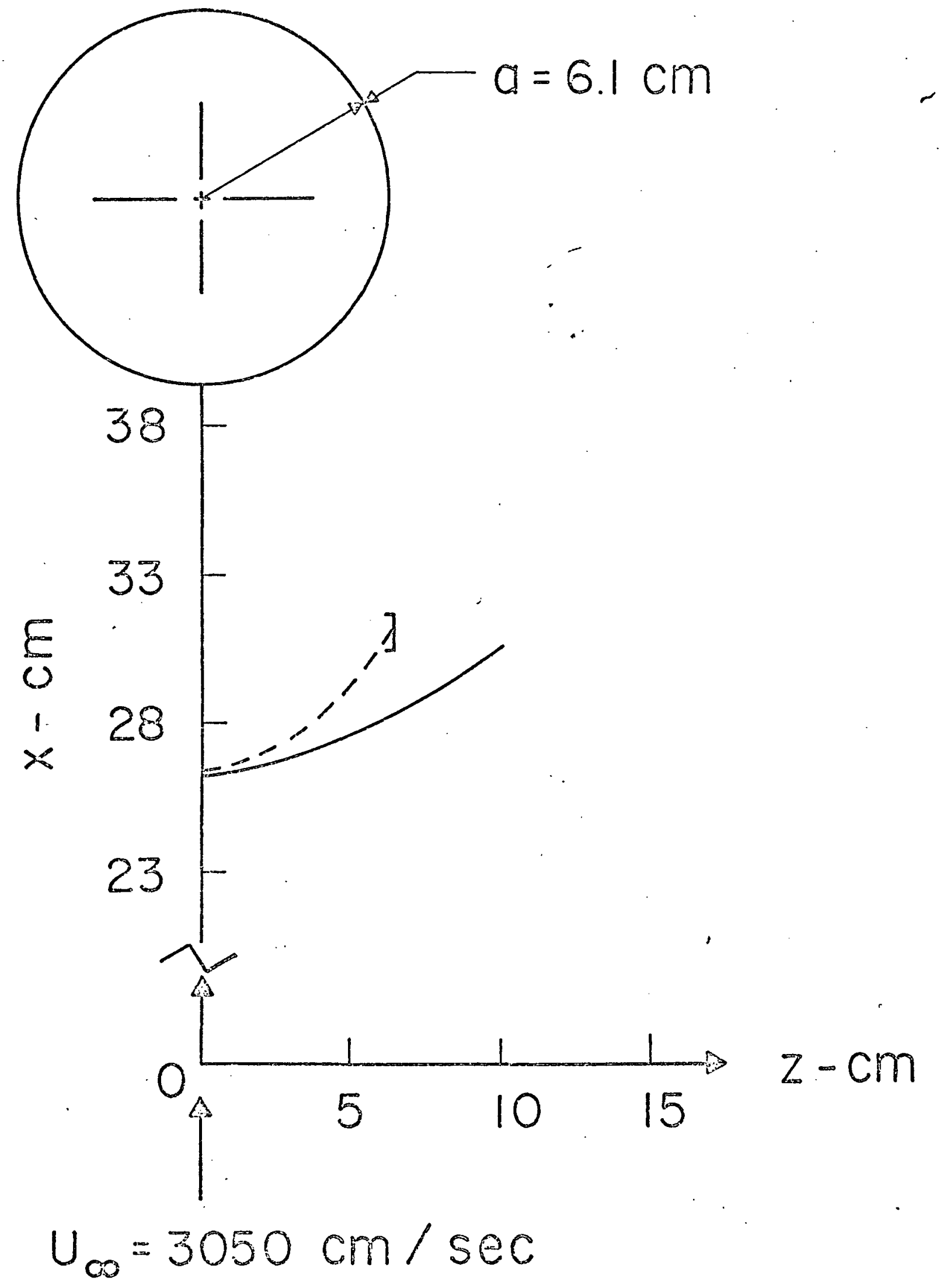


Fig. I Flow geometry and the prediction of flow reversal: --- quasitwo-dimensional (approximate); ___ fully three-dimensional. 